\title{
Comparison between Non-Gaussian Puff Model and a Model Based on a Time-Dependent Solution of Advection-Diffusion Equation
}

\author{
Tiziano Tirabassi ${ }^{1}$, Davidson M. Moreira ${ }^{2}$, Marco Tullio Vilhena ${ }^{3}$, Camila Pinto da Costa ${ }^{4}$ \\ ${ }^{1}$ Institute of Atmospheric Sciences and Climate (ISAC), National Research Council (CNR), Bologna, Italy; ${ }^{2}$ Federal University of \\ Pampa (UNIPAMPA), Bagé, Brazil; ${ }^{3}$ Federal University of Rio Grande do Sul (UFRGS), Porto Alegre, Brazil; ${ }^{4}$ Federal University \\ of Pelotas (UFPel), Pelotas, Brazil. \\ Email: t.tirabassi@isac.cnr.it
}

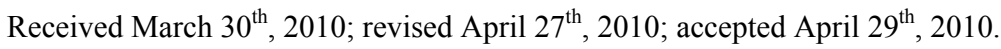

\begin{abstract}
A comparison between a non-Gaussian puff model and an advanced time-dependent model to simulate the pollutant dispersion in the Planetary Boundary Layer is presented. The puff model is based on a general technique for solving the $K$-equation, using the truncated Gram-Charlier expansion (type A) of the concentration field and finite set equations for the corresponding moments. The other model (named ADMM: Analytical Dispersion Multilayers Model) is an semianalytical solution to the time-dependent two-dimensional advection-diffusion equation based on a discretization of the $P B L$ in N sub-layers; in each sub-layers the advection-diffusion equation is solved by the Laplace transform technique, considering an average value for eddy diffusivity and the wind speed. A preliminary performance evaluation is shown in the case of continuous emission from an elevated source in a variable boundary layer. Both models were able to correctly reproduce the concentration field measured and so to be used as operative air pollution models.
\end{abstract}

Keywords: Advection-Diffusion Equation, Air Pollution Modeling, Analytical Solution, Puff Models

\section{Introduction}

Both our scientific understanding and technical developments have been greatly increased by the use of empirical, analytical and numerical models to predict the air pollution concentration in atmosphere. For this purpose, the advection-diffusion equation has been largely applied in operational atmospheric dispersion models. In principle, from this equation it is possible to obtain the dispersion from a source given appropriate boundary and initial conditions plus knowledge of the mean wind velocity and concentration turbulent fluxes [1].

Gaussian models are theoretically based upon an exact, but not realistic solution of the equation of transport and diffusion in the atmosphere, in cases where both wind and turbulent diffusion coefficients are constant with height. The solution is forced to represent real situations by means of empirical parameters, referred to as "sigmas". They can be either stationary (the plume models) or time-dependent (puff models).

Quasi-instantaneous and short-term releases are frequently viewed as "puff" releases. A puff release sce- nario assumes that the release time and sampling times are very short compared to the travel time from the source to the receptor.

The various versions of Gaussian models essentially differ in the techniques utilized to calculate the sigmas as a function of atmospheric stability and the downwind distance from the emission source [2]. Gaussian models are fast, simple and do not require complex meteorological inputs (in comparison to numerical models). For these reasons they are still widely used by the environmental agencies all over the world for regulatory applications.

Nonetheless, because of their well known intrinsic limits, the reliability of a Gaussian model strongly depends on the way the dispersion parameters are determined on the basis of the turbulence structure of the planetary boundary layer (PBL) and the model's ability to reproduce experimental diffusion data. A great variety of formulations exist [3-10]. One of the most popular Gaussian models is AERMOD [11] and among puff models CALPUFF [12] has to be outlined.

Among non-Gaussian models we outline the puff model proposed by van Ulden [13], the plume non- 
Gaussian model by Sharan and Modani [14] and analytical models presented in [15].

In order to take in account the puff releases, Reference [16] developed a K-model for the dispersion of passive non-Gaussian puffs. The model is based on a general technique for solving the advection-diffusion equation using the truncated Gram-Charlier expansion of the concentration field and the finite set of equations for the corresponding moments. Actually, the Gram-Charlier expansion of type A is a classical method for approximating a given distribution with moments of any order, basically consisting of a truncated expansion in terms of Hermite functions, whose coefficients are chosen so as to reproduce the sequence of moments of the distribution up to a given order [17].

Moreover, recently was obtained a semi-analytical solution of the advection-diffusion equation for nonstationary conditions [18]. From this solution a model named ADMM has been set up. The model is based on a discretization of the PBL in $N$ sub-layers, where in each sub-layers the advection-diffusion equation is solved by the Laplace transform technique, considering an average value for eddy diffusivity and the wind speed. The main feature of this method relies on the following steps: stepwise approximation of the eddy diffusivity and wind speed, double Laplace transform application to the advection-diffusion equation in the $x$ and $t$ variable, semi-analytical solution of the set of linear ordinary equation resulting for the double Laplace transform application and construction of the pollutant concentration by the Laplace transform inversion using the Gaussian quadrature scheme (semi-analytical due to the numeric inversion).

The main objective of this paper is to present and discuss the results of a comparison between the nonGaussian puff, where the concentration field is approximated because only the first four moments of the concentration distribution is used, and an advanced time dependent model, that accept general profiles for wind and eddy diffusivity coefficients (as well as the theoretical profiles proposed in the scientific literature, such as the vertical profiles of eddy diffusion coefficients predicted by the Similarity Theory), but they are described by a stepwise function.

\section{The Models}

\subsection{The Puff Model}

The advection diffusion equation describing the time evolution of concentration $C$, due to a release at time $t=0$ of a quantity $Q$ of passive material by an elevated source placed at $(0,0,1)$, in a horizontally homogeneous atmospheric boundary layer is:

$$
\begin{aligned}
& \frac{\partial C}{\partial t}+u \frac{\partial C}{\partial x}+d v \frac{\partial C}{\partial y}=\frac{\partial}{\partial z}\left(K_{z} \frac{\partial C}{\partial z}\right)+ \\
& \quad+K_{h}\left(\frac{1}{d^{2}} \frac{\partial^{2} C}{\partial x^{2}}+\frac{\partial^{2} C}{\partial y^{2}}\right)+\delta(t) \delta(x) \delta(y) \delta(z-1)
\end{aligned}
$$

where $x$ is the along-wind coordinate, $y$ the crosswind one and $z$ the height; $\delta$ means delta function, $(u, v, 0)$ is the wind velocity vector, $K_{z}$ and $K_{h}$ are the eddy diffusivities for vertical and horizontal turbulent transport, respectively. In this chapter all variables are non-dimensional, the corresponding scale factors being given by $H_{s}^{2} / K_{s}$ for time, $U_{s} H_{s}{ }^{2} / K_{s}=d H_{s}$ for a distance along the $x$-axis, $H_{s}$ for the height and distance along the $y$ axis, $K_{s}$ for diffusivities, $u_{s}$ for wind speed and $Q_{0} /\left(d H_{s}^{3}\right)$ for concentration. $K_{s}$ and $u_{s}$ represent the values of the dimensional $u$ and $K$ profiles at the dimensional source $H_{s}$.

The initial condition is:

$$
\lim _{t \rightarrow 0^{+}} \mathrm{C}(\mathrm{x}, \mathrm{y}, \mathrm{z}, \mathrm{t})=0
$$

And the no-flux boundary conditions applied at the ground level and at the mixing layer height $\left(z_{i}\right)$ are:

$$
K_{z} \frac{\partial C}{\partial z}=0 \text { for } z=0 \text { and } z=z_{i}
$$

Since $C$ is exponentially small at asymptotic distances from the source on any horizontal plane, we can introduce the moments of its $(x, y)$-distribution:

$$
C_{m, n}=\int_{-\infty}^{+\infty} \int_{-\infty}^{+\infty} x^{m} y^{n} C d x d y
$$

where $m, n$ are non-negative integers.

Of course, $C_{m, n}$ are functions of height and time.

Their time evolution is governed by the double sequence of 1-dimensional diffusion equations, equivalent to the single three-dimensional (1):

$$
\begin{aligned}
& \frac{\partial C_{0,0}}{\partial t}=D C_{0,0}+\delta(t) \delta(z-1) \\
& \frac{\partial C_{m, n}}{\partial t}=D C_{m, n}+m u C_{m-1, n}+n d v C_{m, n-1}+ \\
& \quad+K_{h}\left(1 / d^{2} m(m-1) C_{m-2, n}+n(n-1) C_{m, n-2}\right)
\end{aligned}
$$

For $m+n \neq 0$ and $D$ the differential operator $\left(\partial / \partial z\left(K_{z} \partial C / \partial z\right)\right)$.

The initial condition is therefore written:

$$
\lim _{t \rightarrow 0^{+}} C_{m, n}=0
$$

And the boundary conditions becomes: 


$$
K_{z} \frac{\partial C}{\partial z}=0 \text { at } z=0, z_{i}
$$

A classic method for approximating a given distribution with moments of any order is the Gram-Chalier expansion of type $\mathrm{A}$, which is basically constituted by a truncated expansion in terms of Hermite functions, whose coefficients are chosen so as to reproduce the sequence of moments of the function up to a given order [19].

In the case of one-variate function of the concentration $C(x)$, truncated to the fourth order, if $S_{k}$ is the skewness and $K_{u}$ is the Kurtosis, we have [16]:

$$
\begin{gathered}
C \cong C_{0} \frac{e^{-\xi^{2} / 2}}{\sigma \sqrt{2 \pi}} \\
\cdot\left[1+\left(\frac{K_{u}-3}{24}\right)\left(\xi^{4}-6 \xi^{2}+3\right)+\frac{S_{k}}{6} \xi\left(\xi^{2}-3\right)\right]
\end{gathered}
$$

where:

$$
\begin{gathered}
\xi=\frac{x-b}{\sigma} \\
b=\frac{C_{1}}{C_{0}} \\
\sigma^{2}=\frac{C_{2}}{C_{0}}-b^{2} \\
S_{k}=\frac{1}{\sigma^{3}}\left[\frac{C_{3}}{C_{0}}-3 b \sigma^{2}-b^{3}\right] \\
K_{u}=\frac{1}{\sigma^{4}}\left[\frac{C_{4}}{C_{0}}-6 b^{2} \sigma^{2}-4 b \sigma^{3} S_{k}-b^{4}\right]
\end{gathered}
$$

\subsection{ADMM Model}

A typical problem in air pollution studies is to seek the solution for the cross-wind ( $y$ direction) integrated concentration for a continuous source of pollution (being lateral concentration distribution usually assumed Gaussian), that is:

$$
\frac{\partial \bar{C}}{\partial t}+u \frac{\partial \bar{C}}{\partial x}=\frac{\partial}{\partial z}\left(K_{z} \frac{\partial \bar{C}}{\partial z}\right)
$$

where $\bar{C}$ is the dimensional cross-wind integrated concentration (from this section all the variable are dimensional).

The mathematical description of the dispersion problem represented by the (9) is well posed when it is provided by initial and boundary conditions. Indeed, it is assumed that at the beginning of the contaminant release the dispersion region is not polluted, this means:

$$
\bar{C}(x, z, 0)=0 \quad \text { at } \quad t=0
$$

At the point $\left(0, H_{s}, t\right)$ a continuous line source of the constant emission rate $Q$ is assumed

$$
\bar{C}(0, z, t)=\frac{Q}{u} \delta\left(z-H_{s}\right) \quad \text { at } \quad x=0
$$

where $\delta$ is the Dirac delta function and $H_{s}$ the source height.

The pollutants are also subjected to the boundary conditions of zero flux at ground and PBL top:

$$
K_{z} \frac{\partial \bar{C}}{\partial z}=0 \quad \text { at } \quad z=0, z_{i}
$$

where $z_{i}$ is the vertical depth of mixing region (PBL height).

Bearing in mind the dependence of the $K_{z}$ coefficient and wind speed profile $u$ on variable $z$, the height $z_{i}$ of a PBL is discretized in N sub-intervals in such a manner that inside each interval $K_{z}$ and $u$ assume the average value:

$$
\begin{gathered}
K_{n}=\frac{1}{z_{n+1}-z_{n}} \int_{z_{n}}^{z_{n+1}} K_{z}(z) d z \\
u_{n}=\frac{1}{z_{n+1}-z_{n}} \int_{z_{n}}^{z_{n+1}} u(z) d z
\end{gathered}
$$

Therefore the solution of (9) is reduced to the solution of $\mathrm{N}$ problems of the type:

$$
\frac{\partial \bar{C}_{n}}{\partial t}+u_{n} \frac{\partial \bar{C}_{n}}{\partial x}=K_{n} \frac{\partial^{2} \bar{C}_{n}}{\partial z^{2}} \quad z_{n} \leq z \leq z_{n+1}
$$

for $n=1, \ldots, N-1$, where $\bar{C}=\overline{C_{n}}$ denotes the concentration at the $n^{\text {th }}$ sub-interval. To determine the $2 N$ integration constants the additional $(2 N-2)$ condition namely continuity of concentration and flux at interface are considered:

$$
\begin{gathered}
\bar{C}_{n}=\bar{C}_{n+1} \quad n=1,2, \ldots(N-1) \\
K_{n} \frac{\partial \bar{C}_{n}}{\partial z}=K_{n+1} \frac{\partial \bar{C}_{n+1}}{\partial z} \quad n=1,2, \ldots(N-1)
\end{gathered}
$$

Applying the Laplace transform in (15) results:

$\frac{\partial^{2}}{\partial z^{2}} \overline{\bar{C}}_{n}(s, z, p)-\frac{\left(p+s u_{n}\right)}{K_{n}} \overline{\bar{C}}_{n}(s, z, p)=-\frac{u_{n}}{K_{n}} \bar{C}_{n}(0, z, p)$

where $\overline{\bar{C}}_{n}(s, z, p)=L\left\{\bar{C}_{n}(x, z, t) ; x \rightarrow s ; t \rightarrow p\right\}$, which has the well-know solution:

$$
\begin{aligned}
\overline{\bar{C}}_{n}(s, z, p)=A_{n} e^{-R_{n} z}+B_{n} e^{R_{n} z}+ \\
+\frac{Q}{2 R_{a}}\left(e^{-R_{n}\left(z-H_{s}\right.}-e^{R_{n}\left(z-H_{s}\right)}\right)
\end{aligned}
$$

where 


$$
R_{n}=\sqrt{\frac{\left(p+s u_{n}\right)}{K_{n}}} \text { and } R_{a}=\sqrt{\left(p+s u_{n}\right) K_{n}}
$$

Finally, applying the initial and boundary conditions we get a linear system for the integration constants. Henceforth the concentration is obtained by inverting numerically the transformed concentration $\overline{\bar{C}}_{n}$ by Gaussian quadrature scheme [18]:

$$
\begin{aligned}
& \bar{C}(x, z, t)=\sum_{i=1}^{k} w_{i}\left(\frac{P_{i}}{t}\right) \sum_{J=1}^{M} w_{j}\left(\frac{P_{j}}{x}\right)
\end{aligned}
$$

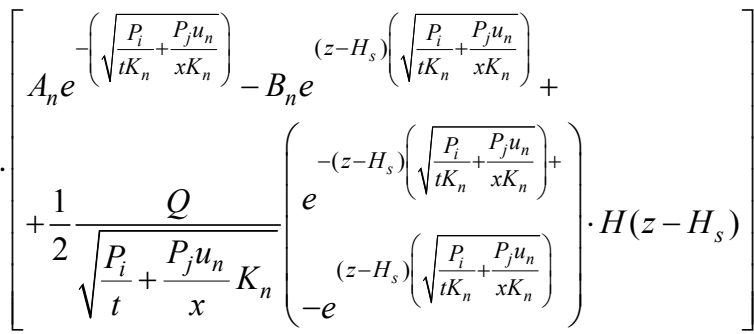

where $H\left(z-H_{s}\right)$ is the Heaviside function that multiplies the part of (20) that is different from 0 only in the sublayer that contains the source. The values of $w_{i}, w_{j}$ (weights) and $P_{i}, P_{j}$ (roots) of the Gaussian quadrature scheme are tabulated in the book by Stroud and Secrest [20] and $k$ and $m$ are the quadrature points.

The semi-analytical character of the solution (20) reduces to the solution of [21] when the time goes to infinity $(t \rightarrow \infty)$. For more details see [22-24].

\section{Boundary Layer Parameterization}

We applied a parameterization proposed by Troen and Marth [25], as presented in Pleim and Chang [26]. During near neutral and stable conditions $\left(z_{i} / L \geq-10\right)$, we adopted:

$$
K_{z}=\frac{k u_{*} z\left(1-z / z_{i}\right)^{2}}{\Phi_{h}(z / L)}
$$

where $\Phi_{h}=(1-16 z / L)^{-1 / 2}$.

During convective conditions $\left(z_{i} / L<-10\right)$ the friction velocity was replaced by the convective velocity $\left(w_{*}\right)$ as scaling velocity, to give [26]:

$$
K_{z}=k w_{*} z\left(1-z / z_{i}\right)
$$

where $k$ is the von Karman constant $(k \sim 0.4)$.

For the horizontal eddy diffusivity in unstable conditions (in the puff model), we use [27]:

$$
K_{h}=0.1 w_{*} z_{i}
$$

In neutral-stable conditions [28]:

$$
K_{h}=2 K_{M z}
$$

where $K_{M z}$ is the maximum of $K_{z}$.

\section{Validation against Experimental Data}

For a better understanding of the comparison between the two models in this work, we focus our attention on the mathematical features of the two approaches. Concerning the semi-analytical method it is relevant to underline that in this approach no approximation is made in its derivation except for the stepwise approximation of the meteorological parameters and the Laplace numerical inversion by the Gaussian quadrature scheme. It is well known, that the results attained by the Gaussian quadrature scheme of order $N$, are exact when the transformed function is a polynomial of degree $(2 N-1)$. It is possible, performing relative error calculation, between numerical results with $k+1$ and $k$ points of quadrature as well for $m+1$ and $m$ points, to control the error in the Gaussian quadrature scheme, by properly choosing $k$ and $m$, in order to attain a prescribed accuracy. Concerning the issue of stepwise approximation, it is important to bear in mind that the stepwise approximation of a continuous function converges to the continuous function, when the stepwise of the approximation goes to zero. For the semi-analytical method it is only necessary to choose the number of the sub-layers in an appropriate manner, by taking the smoothness of the functions $K_{z}$ and $u$ into account.

On the other hand regarding the puff model, derivatives of the resulting system of equations are approximated by a finite difference scheme, which does not occur in the semi-analytical method. According to the Lax equivalence theorem [29] for linear autonomous problems, the convergence of the numerical schemes demands the fulfillment of the requirements of stability and consistency. In order to achieve the stability conditions, the numerical methods like the one considered in this work, impose a large number of step calculations used in performing the time integration for all times. This feature does not appear in the semi-analytical method, because the semi-analytical character of the solution allows us to perform the calculation at any time. This guarantees the smaller computational time as well the smaller round-off error influence in the accuracy of the results of the semi-analytical method when compared with the numerical ones, because it demands less mathematical operations. Moreover the concentration field is described only using the first four moments of the concentration distribution. In fact we use the Gram-Charlier expansion (type A) of the concentration field truncated at the fourth moment.

A preliminary evaluation of the performances of the 
two models (with the boundary layer parameterization proposed) is presented, using the Copenhagen data set [30]. The Copenhagen data set is composed of tracer SF6 data from dispersion experiments carried out in northern Copenhagen. The tracer was released without buoyancy from a tower at a height of $115 \mathrm{~m}$ and was collected at ground-level positions in up to three crosswind arcs of tracer sampling units. The sampling units were positioned 2-6 km far from the point of release. We used the values of the crosswind-integrated concentrations normalized with the tracer release rate from [31]. Tracer releases typically started up 1 hour before the tracer sampling and stopped at the end of the sampling period. The site was mainly residential with a roughness length of $0.6 \mathrm{~m}$. Generally the distributed data set contains hourly mean values of concentrations and meteorological data. However, in this work, we used data with a greater time resolution kindly made available by Gryning [32]. In particular, we used 20 minutes averaged measured concentrations and 10 minutes averaged values for meteorological data.

Tables 1-3 report the friction velocity, the Monin-Obukhov length and boundary layer height (only one value for each run), respectively, used in the simulations.

In Figure 1 the calculated concentrations are plotted against the measured ones for the two approaches.

Table 4 presents some statistical indices, defined as normalised mean square error (nmse), correlation coefficient (r), factor of two (fa2), fractional bias (fb) and fractional standard deviation (fs) [33]:

$$
\begin{gathered}
\mathrm{nmse}=\frac{\overline{\left(C_{o}-C_{p}\right)^{2}}}{\overline{C_{o} \overline{C_{p}}}} \\
\mathrm{r}=\frac{\overline{\left(C_{o}-\overline{C_{o}}\right)\left(C_{p}-\overline{C_{p}}\right)}}{\sigma_{o} \sigma_{p}}
\end{gathered}
$$

fa2 = data for which $0.5 \leq C_{p} / C_{o} \leq 2$

Table 1. Friction velocitiy $(\mathrm{m} / \mathrm{s})$ for the different runs and time steps. Every time step corresponds at 10 minutes

\begin{tabular}{ccccccccc}
\hline Run & 1 & 2 & 3 & 4 & 5 & 7 & 8 & 9 \\
\hline 1 & 0.36 & 0.68 & 0.46 & 0.56 & 0.58 & 0.48 & 0.65 & 0.72 \\
2 & 0.37 & 0.67 & 0.45 & 0.51 & 0.52 & 0.48 & 0.79 & 0.73 \\
3 & 0.40 & 0.81 & 0.47 & 0.37 & 0.51 & 0.57 & 0.67 & 0.60 \\
4 & 0.43 & 0.68 & 0.39 & 0.44 & 0.58 & 0.62 & 0.67 & 0.59 \\
5 & 0.35 & 0.75 & 0.39 & 0.48 & 0.59 & 0.53 & 0.68 & 0.65 \\
6 & 0.34 & 0.74 & 0.40 & 0.48 & 0.52 & 0.65 & 0.65 & 0.71 \\
7 & 0.42 & 0.76 & 0.40 & 0.39 & 0.52 & 0.63 & 0.68 & 0.73 \\
8 & 0.43 & 0.82 & 0.41 & 0.40 & 0.45 & 0.65 & 0.67 & 0.73 \\
9 & 0.40 & 0.76 & 0.31 & 0.39 & 0.44 & 0.66 & 0.73 & 0.73 \\
10 & 0.37 & 0.73 & 0.34 & 0.39 & 0.44 & 0.62 & 0.73 & 0.66 \\
11 & 0.35 & 0.69 & 0.39 & 0.39 & 0.44 & 0.52 & 0.75 & 0.67 \\
12 & 0.36 & 0.66 & 0.40 & 0.39 & 0.43 & 0.62 & 0.69 & 0.74 \\
\hline
\end{tabular}

Table 2. Monin-Obukhov length (m) for the different runs and time steps. Every time step corresponds at $\mathbf{1 0}$ minutes

\begin{tabular}{ccccccccc}
\hline $\boldsymbol{R} \backslash \mathbf{n}$ & 1 & 2 & 3 & 4 & 5 & 7 & 8 & 9 \\
\hline 1 & -26 & -178 & -152 & -75 & -492 & -71 & -71 & -793 \\
2 & -23 & -227 & -194 & -42 & -215 & -80 & -85 & -471 \\
3 & -83 & -311 & -106 & -23 & -368 & -64 & -47 & -202 \\
4 & -42 & -160 & -101 & -32 & -735 & -111 & -49 & -366 \\
5 & -36 & -203 & -129 & -71 & -366 & -177 & -45 & -633 \\
6 & -42 & -286 & -70 & -80 & -273 & -67 & -63 & -13588 \\
7 & -47 & -155 & -83 & -83 & -273 & -87 & -41 & -593 \\
8 & -38 & -228 & -60 & -101 & -262 & -71 & -47 & -471 \\
9 & -83 & -184 & -106 & -129 & -395 & -56 & -70 & -389 \\
10 & -21 & -389 & -42 & -129 & -395 & -111 & -64 & -375 \\
11 & -32 & -133 & -101 & -129 & -395 & -215 & -52 & -262 \\
12 & -29 & -375 & -70 & -129 & -759 & -123 & -39 & -252 \\
\hline
\end{tabular}

Table 3. Boundary layer height for the different runs

\begin{tabular}{ccccccccc}
\hline Run & 1 & 2 & 3 & 4 & 5 & 7 & 8 & 9 \\
\hline $\boldsymbol{z}_{\boldsymbol{i}}$ & 1980 & 1920 & 1120 & 390 & 820 & 1850 & 810 & 2090 \\
\hline
\end{tabular}

Table 4. Statistical evaluation of models results. Model 1 is the puff model and 2 ADMM model

\begin{tabular}{cccccc}
\hline model & nmse & $\mathrm{r}$ & $\mathrm{fa} 2$ & $\mathrm{fb}$ & $\mathrm{fs}$ \\
\hline 1 & 0.21 & 0.74 & 0.90 & 0.10 & 0.45 \\
2 & 0.15 & 0.81 & 0.95 & 0.18 & 0.38 \\
\hline
\end{tabular}

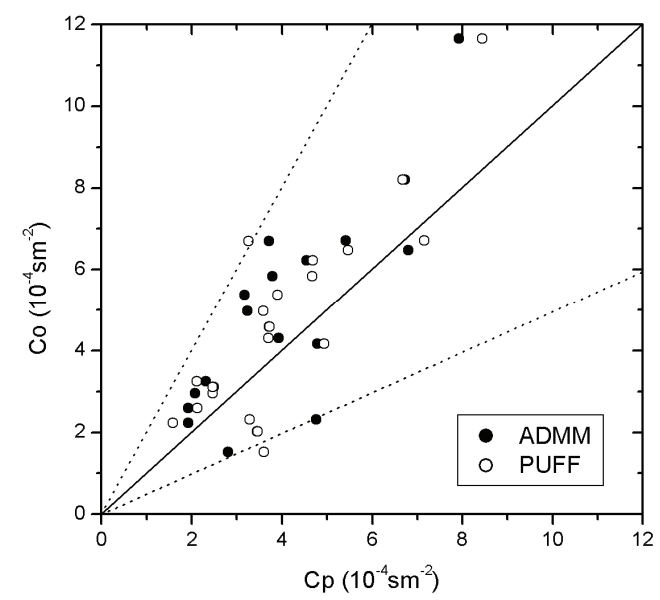

Figure 1. Scatter plot of observed (Co) versus predicted (Cp) crosswind-integrated concentrations normalized with the emission source rate. Points between dashed lines are in a factor of two

$$
\mathrm{fb}=2 \frac{\overline{C_{o}}-\overline{C_{p}}}{\overline{C_{o}}+\overline{C_{p}}}
$$




$$
\mathrm{fs}=2 \frac{\sigma_{o}-\sigma_{p}}{\sigma_{o}+\sigma_{p}}
$$

where the subscript " $o$ " and " $p$ " are for the observed and predicted concentrations, respectively, while $\sigma$ is the standard deviation.

Analysing the statistical indices in Table 4 it is possible to notice that the models simulate satisfactorily the observed concentrations, with nmse, fb and fs values relatively near to zero and $\mathrm{r}$ and fa2 relatively near to 1 . A more detailed inspection of the Table 4 permits to observe that ADMM presents best values of nmse, $r$ and fa2 while the puff model presents best values of $\mathrm{fb}$.

\section{Conclusions}

The aim of this paper was to present and discuss the results of a comparison between non-Gaussian puff model and a model based on a time-dependent semy-analytical solution of advection-diffusion equation to simulate pollutant dispersion in the PBL, focusing the ability to correctly reproduce the concentration field measured during the Copenhagen tracer experiments, considering the meteorological parameters varying with time.

Models performances were evaluated using data from the Copenhagen data set, but with a time resolution greater than the data ones generally distributed [34], presenting good results for both approaches. In particular, we used 20 minutes average concentrations of SF6 and 10 minutes average values for meteorological data.

The two models present comparable results and, in this preliminary evaluation, their performance does not show any correlation with the meteorological characterization of the PBL. Both models underestimate the ground level concentrations, probably due to the turbulence parameterization used.

\section{Acknowledgements}

The authors are gratefully indebted to CNPq, FAPERGS, CNR and ENVIREN for the partial financial support of this work.

\section{REFERENCES}

[1] S. P. Arya, "Air Pollution Meteorology and Dispersion," Oxford University Press, Oxford, 1999, p. 310.

[2] P. Zannetti, "Air Quality Modeling III," The EnvironComp Institute and Air \& Waste Management Association, Fremont, 2008, p. 485.

[3] S. R. Hanna, G. A. Briggs, J. Deardoff, B. A. Egan, F. A. Gifford and F. Pasquill, "AMS Workshop on Stability Classification Schemes and Sigmas Curves-Summary of Recommendations," Bulletin of the American Meteorological Society, Vol. 58, No. 12, 1977, pp. 1305-1309.

[4] G. A. Briggs, "Plume Rise Predictions," Lectures on Air
Pollution and Environmental Impact Analyses, Workshop Proceedings, American Meteorological Society, Boston, 29 September-3 October 1975, pp. 59-111.

[5] S. R. Hanna, "Lateral Dispersion from Tall Stacks," Journal of Climmate Applied Meteorology, Vol. 25, No. 10, 1986, pp. 1426-1433.

[6] R. Berkowicz, H. R. Olesen and U. Torp, "The Danish Gaussian Air Pollution Model (OML): Description, Test and Sensivity Analysis in View of Regulatory Applications," In: C. De Wispelaere, F. A. Schiermeier and N. V. Gillani, Eds., Proceedings of NATO-CCMS 16th International Meeting on Air Pollution, Modelling and its Applications, Plenum Press, New York, 1986, pp. 453-481.

[7] B. M. Bowen, "Long-Term Tracer Study at Los Alamos, New Messico. Part II: Evaluation and Comparison of Several Methods to Determinate Dispersion Coefficients," Journal of Appied Meteorology, Vol. 33, No. 11, 1994, pp. 1236-1254.

[8] J. J. Erbrink, "Use of Boundary-Layer Meteorological Parameters in the Gaussian Model 'Stacks'," BounaryLayer Meteorology, Vol. 74, No. 3, 1995, pp. 211-235.

[9] M. Mohan and T. A. Siddiqui, "An Evaluation of Dispersion Coefficients for Use in Air Quality Models," BounaryLayer Meteorology, Vol. 84, No. 2, 1997, pp. 177-205.

[10] B. J. Tsuang, "Quantification on the Source/Receptor Relationship of Primari Pollutants and Secondary Aerosols by a Gaussian Plume Trajectory Model: Part I - Theory," Atmospheric Environment, Vol. 37, No. 28, 2003, pp. 39813991.

[11] A. J. Cimorelli, S. G. Perry, A. Venkatram, J. C. Weil, R. J. Paine, R. B. Wilson, R. F. Lee, W. D. Peters and R. W. Brode, "AERMOD: A Dispersion Model for Industrial Source Applications. Part I: General Model Formulation and Boundary Layer Characterization," Journal of Appied Meteorology, Vol. 44, No. 5, 2005, pp. 682-693.

[12] J. S. Scire, D. G. Strimaitis and R. J. Yamartino, "A User's Guide for the CALPUFF Dispersion Model," Version 5, Earth Tech Inc., Lowell, 2000. http://www.src. com/calpuff/calpuff1.htm

[13] A. P. van Ulden, "A Surface-Layer Similarity Model for the Dispersion of a Skewed Passive Puff near the Ground," Atmospheric Environment, Vol. 26, No. 4, 1992, pp. 681692.

[14] M. Sharan and M. Modani, "A Two-Dimensional Analytical Model for the Dispersion of Air-Pollutants in the Atmosphere with a Capping Inversion," Atmospheric Environment, Vol. 40, No. 19, 2006, pp. 3479-3489.

[15] T. Tirabassi, "Operational Advanced Air Pollution Modeling," Pure and Applied Geophysics, Vol. 160, No. 1-2, 2003, pp. 5-16.

[16] R. Lupini and T. Tirabassi, "Solution of the AdvectionDiffusion Equation by the Moments Method," Atmospheric Environment, Vol. 17, No. 5, 1983, pp. 965-971.

[17] T. Tirabassi and U. Rizza, "Boundary Layer Parameterization for a Non-Gaussian Puff Model," Journal of Appied Meteorology, Vol. 36, No. 8, 1997, pp. 1031-1037. 
[18] D. M. Moreira, P. V. F. Neto and J. C. Carvalho, "Analytical Solution of the Eulerian Dispersion Equation for Nonstationary Conditions: Development and Evaluation," Environmental Modelling and Software, Vol. 20, No. 9, 2005, pp. 1159-1165.

[19] M. Kendall and A. Stuart, "The Advanced Theory of Statistic," Charles Griffin, London, 1977, pp. 1-472.

[20] A. H. Stroud and D. Secrest, "Gaussian Quadrature Formulas,” Prentice-Hall, Englewood Cliffs, 1996, p. 320.

[21] D. M. Moreira, G. A. Degrazia and M. T. Vilhena, "Dispersion from Low Sources in a Convective Boundary Layer: An Analytical Model," Il Nuovo Cimento, Vol. 22C, No. 5, 1999, pp. 685-691.

[22] C. Mangia, D. M. Moreira, I. Schipa, G. A. Degrazia, T. Tirabassi and U. Rizza, "Evaluation of a New Eddy Diffusivity Parameterisation from Turbulent Eulerian Spectra in Different Stability Conditions," Atmospheric Environment, Vol. 36, No. 1, 2002, pp. 67-76.

[23] D. M. Moreira, M. T. Vilhena, J. C. Carvalho and G. A. Degrazia, "Analytical Solution of the Advection-Diffusion Equation with Nonlocal Closure of the Turbulent Diffusion," Environmental Modelling and Software, Vol. 20, No. 10, 2004, pp. 1347-1351.

[24] C. P. Costa, M. T. Vilhena, D. M. Moreira and T. Tirabassi, "Semi-Analytical Solution of the Steady ThreeDimensional Advection-Diffusion Equation in the Planetari Boundary Layer," Atmospheric Environment, Vol. 40, 2006, pp. 5659-5669.

[25] I. Troen and L. Marth, "A Simple Model of the Atmospheric Boundary Layer; Sensitivity to Surface Evaporation," Bounary-Layer Meteorology, Vol. 37, No. 1-2, 1986, pp. 129-148.

[26] J. Pleim and J. S. Chang, "A Non-Local Closure Model for Vertical Mixing in the Convective Boundary Layer,"
Atmospheric Environment, Vol. 26, No. 6, 1992, pp. $965-$ 981.

[27] J. H. Seinfeld and S. N. Pandis, "Atmospheric Chemistry and Physics," John Wiley \& Sons, New York, 1998, p. 1326.

[28] G. Tangerman, "Numerical Simulations of Air Pollutant Dispersion in a Stratified Planetary Boundary Layer," Atmospheric Environment, Vol. 12, No. 6-7, 1978, pp. 13651369.

[29] P. K. Kythe, P. Puri and M. R. Schäferkotter, "Partial Differential Equations and Mathematics," CRC Press, Boca Raton, 2002, p. 440.

[30] S. E. Gryning and E. Lyck, "Atmospheric Dispersion from Elevated Sources in an Urban Area: Comparison between Tracer Experiments and Model Calculations," Journal of Climate and Applied Meteorology, Vol. 23, No. 4, 1984, pp. 651-660.

[31] S. E. Gryning, A. A. M. Holtslag, J. S. Irwin and B. Siversten, "Applied Dispersion Modelling Based on Meteorological Scaling Parameters," Atmospheric Environment, Vol. 21, No. 1, 1987, pp. 79-89.

[32] D. M. Moreira, M. T. Vilhena, D. Buske and T. Tirabassi, "The GILTT Solution of the Advection-Diffusion Equation for an Inhomogeneous and Nonstationary PBL," Atmospheric Environment, Vol. 40, No. 17, 2006, pp. 31863194.

[33] S. R. Hanna, "Confidence Limit for Air Quality Models as Estimated by Bootstrap and Jacknife Resampling Methods," Atmospheric Environment, Vol. 23, No. 6, 1989, pp. 1385-1395.

[34] H. R. Olesen, "Datasets and Protocol for Model Validation," International Journal of Environment and Pollution, Vol. 5, No. 4-6, 1995, pp. 693-701. 